\title{
Highly efficient electrocatalytic hydrogen evolution reaction on carbonized porous conducting polymers
}

\author{
Abhishek Lahiri ${ }^{1,2} \cdot$ Guozhu Li $^{1} \cdot$ Frank Endres $^{1}$ \\ Received: 8 March 2020 / Revised: 25 March 2020 / Accepted: 25 March 2020 / Published online: 12 April 2020 \\ (C) The Author(s) 2020
}

\begin{abstract}
A rational design of an efficient and inexpensive electrocatalyst for water splitting still remains a challenge. Porous conducting polymers are attractive materials which not only provide a high surface area for electrocatalysis but also absorb light which can be harnessed in photoelectrocatalysis. Here, a novel and inexpensive electrochemical approach is developed to obtain nanoporous conducting copolymers with tunable light absorbance and porosity. By fine-tuning the copolymer composition and upon heat treatment, an excellent electrocatalytic hydrogen evolution reaction (HER) was achieved in alkaline solution with an overpotential of just $77 \mathrm{mV}$ to obtain a current density of $10 \mathrm{~mA} \mathrm{~cm}{ }^{-2}$. Such an overpotential is remarkably low compared with other reported values for polymers in an alkaline medium. The nanoporous copolymer developed here shows a great promise of using metal-free electrocatalysts and brings about new avenues for exploitation of these porous conducting polymers.
\end{abstract}

Keywords Porous polymers $\cdot$ Metal-free electrocatalysts $\cdot$ Hydrogen evolution reaction $\cdot$ Electrocatalysis $\cdot$ Ionic liquids · Conducting polymers

\section{Introduction}

Electrocatalytic water splitting is a lucrative process for generating sustainable hydrogen as a fuel. Although a minimum thermodynamic electrode potential of $1.23 \mathrm{~V}$ is needed to split water, with the hydrogen production occurring at $0 \mathrm{~V}$ vs standard hydrogen electrode (SHE), only by the use of noble metal catalysts such as Pt and its alloys a low overvoltage close to theoretical value with a high exchange current density and a small Tafel slope is achievable [1-3]. Considerable search for other non-noble metals and metal oxides is ongoing to develop a cost-effective alternate catalyst for hydrogen production.

Electronic supplementary material The online version of this article (https://doi.org/10.1007/s10008-020-04577-3) contains supplementary material, which is available to authorized users.

Abhishek Lahiri

abhishek.lahiri@brunel.ac.uk

Frank Endres

frank.endres@tu-clausthal.de

1 Institute of Electrochemistry, Clausthal University of Technology, Arnold-Sommerfeld-Strasse 6, 38678 Clausthal-Zellerfeld, Germany

2 Department of Chemical Engineering, Brunel University London, Uxbridge UB8 3PH, UK
In the last two decades, various 3D transition metals such as $\mathrm{Co}, \mathrm{Fe}, \mathrm{Ni}$, and their alloys, along with their phosphide and sulfide compounds, have shown promising results for HER with low overvoltage [4-6]. However, they are susceptible to corrosion in both acidic and alkaline environments.

Recently, carbon-based materials have shown to be promising electrodes for photocatalytic and electrocatalytic hydrogen production [7]. These metal-free electrocatalysts provide a cheaper approach for hydrogen evolution reaction as they are abundant and also are tolerant to both acidic and alkaline environments. Their low dimensional structures have shown to be promising materials for oxygen reduction and evolution reactions. Very recently, Zheng et al. showed that carbon nitride combined with graphene gave an overpotential of $240 \mathrm{mV}$ for HER in acidic solution to achieve a current density of $10 \mathrm{~mA} \mathrm{~cm}^{-2}$ [8]. Using density functional theory calculations, they predicted that due to chemical coupling of carbon nitride and graphene, a decrease in energy barrier compared to carbon materials was achieved for HER to take place. Subsequent research on developing 2D-based carbon materials and their hybrids with transition metals are being explored for HER [9]. However, during the synthesis of such materials and its testing for HER, certain drawbacks occur such as inevitable aggregation of carbon or carbon/metal hybrids, poor contact between carbon and electrodes, and 
formation of thick carbon layers which decrease the porosity and lead to lower HER efficiency.

On the other hand, organic semiconductors including porous polymeric carbonaceous materials have been reported to be better suited for HER applications [10, 11]. The semiconducting property of polymeric materials makes it an attractive option for photoelectrochemical hydrogen production by tuning the highest occupied molecular orbital (HOMO) and lowest unoccupied molecular orbital (LUMO) levels [11]. Besides HOMO/LUMO, the porosity also plays a critical role for water splitting by providing more active catalytic sites and by decreasing the hydrogen buildup on the catalyst and therefore minimizes the concentration overpotential for HER [12].

Organic semiconductors including conducting polymers have mainly been synthesized by hydrothermal or other solution/pyrolysis routes. Electrodeposition of organic semiconductors has been shown using organic solvents and ionic liquids [13-15]. In ionic liquids, as it is possible to change the cation/anion combination, structural changes in the polymeric structure are possible [15]. The polymerization of benzene to polyparaphenylene (PPP) has been shown from 1-hexyl-3methylimidazolium tris(pentafluoroethyl)trifluorophosphate ([HMIM]FAP) [16]. However, the functional properties were not tested. Furthermore, attempts to make PPP based co-polymers from ionic liquids have not yet been shown.

Taking these factors into consideration, in this manuscript, we demonstrate an electrochemical synthesis of porous copolymers based on polythiophene (PTh) and polyparaphenylene (PPP) from an ionic liquid. On electrochemical copolymerization from ionic liquids, hierarchical porous structures could be directly obtained without any template-assisted techniques [13]. The HER on the electrodeposited porous polymer electrodes showed promising results in an alkaline medium. A significant improvement in the HER catalytic property was obtained on carbonizing the polymers.

\section{Experimental section}

$1-\mathrm{H}$ e x y $1-3-\mathrm{m}$ e th y $1 \mathrm{im}$ i d a z o 1 i u m tris(pentafluoroethyl)trifluorophosphate (HMIMFAP) was purchased in the highest available quality from Merck (Germany) and was used after drying under vacuum at $100{ }^{\circ} \mathrm{C}$ to remove the water content to below $2 \mathrm{ppm}$. Benzene (99.8\%) and thiophene (>99\%) were obtained from Sigma. A copper plate was used as the working electrode which was cleaned in a mixture of acetone and isopropanol in an ultrasonic bath prior to the experiments. Platinum wires were used as counter and quasi-reference electrodes (QRE) which were heated in a hydrogen flame to remove any surface impurities. The electrochemical cell was made of PTFE and clamped over a PTFE-covered Viton ${ }^{\circledR}$ O-ring onto the substrate, providing a geometric area of $0.3 \mathrm{~cm}^{2}$. The Teflon cell and the O-ring were cleaned in a mixture of 50:50 vol\% of concentrated $\mathrm{H}_{2} \mathrm{SO}_{4}$ and $\mathrm{H}_{2} \mathrm{O}_{2}(35 \%)$ followed by refluxing in distilled water.

The electrochemical measurements were performed in an argon-filled glove box with water and oxygen contents of below $0.5 \mathrm{ppm}$ (OMNI-LAB from Vacuum Atmospheres) by using a VersaStat II (Princeton Applied Research) potentiostat/galvanostat controlled by PowerSuite ${ }^{\circledR}$ software. The scan rate during cyclic voltammetry $(\mathrm{CV})$ was set to $10 \mathrm{mV} \mathrm{sec}{ }^{-1}$. For polymer deposition, $\mathrm{CV}$ was performed for 10 cycles between the open circuit potential and $2.3 \mathrm{~V}$ vs $\mathrm{Pt}$ on $\mathrm{Cu}$ from HMIMFAP-based solutions: $0.2 \mathrm{M}$ thiophene (pure $\mathrm{PTh}$ ), $0.15 \mathrm{M}$ thiophene $+0.05 \mathrm{M}$ benzene, $0.1 \mathrm{M}$ thiophene $+0.1 \mathrm{M}$ benzene, $0.05 \mathrm{M}$ thiophene $+0.15 \mathrm{M}$ benzene, and $0.2 \mathrm{M}$ benzene (pure PPP).

For hydrogen evolution reaction, a three-electrode setup was used with the conducting polymer as the working electrode, saturated calomel electrode (SCE) as the reference electrode, and carbon as the counter electrode. The electrolyte was $1 \mathrm{M} \mathrm{KOH}$. Prior to the experiments, the electrolyte was deaerated with $\mathrm{N}_{2}$ for $30 \mathrm{~min}$. The polarization curves were recorded by applying a scan rate of $10 \mathrm{mV} \mathrm{s}^{-1}$. The effect of visible light on the HER was evaluated by illuminating the working electrode with a regular LED torch (Powerlite). The potentials were not iR compensated. The potentials were converted to a reversible hydrogen electrode (RHE) scale using $\mathrm{E}(\mathrm{RHE})=0.241+0.059 \times \mathrm{pH}$. For heat treatment, the polymers were put inside a tube furnace with continuous Ar flow and heated at $700{ }^{\circ} \mathrm{C}$ for $1 \mathrm{~h}$. Ten percent of experiments did show some peeling off the heat-treated polymer from the copper substrate. However, most of the deposit showed good adhesion on copper. For scanning electron microscopy (SEM) (JEOL JSM7610F) and energy dispersive X-ray (EDX) analyses, the electrodes were cleaned with isopropanol overnight before the measurement.

X-ray photoelectron spectra (XPS) were obtained using an ultrahigh vacuum (UHV) apparatus with a base pressure below $1 \times 10^{-10} \mathrm{hPa}$. The sample was irradiated using the $\mathrm{Al} \mathrm{K} \alpha$ line (photon energy of $1486.6 \mathrm{eV}$ ) of a non-monochromatic X-ray source (Omicron DAR 400). Electrons emitted were detected by a hemispherical analyzer (Omicron EA125) under an angle of $45^{\circ}$ normal to the surface with a resolution of $0.83 \mathrm{eV}$ for detail spectra and $2.07 \mathrm{eV}$ for survey spectra, respectively. All XPS spectra were displayed as a function of the binding energy with respect to the Fermi level.

Fourier transform infrared spectroscopy (VERTEX $70 \mathrm{~V}$, Bruker Optics $\mathrm{GmbH}$ ) with an attached attenuated total reflectance (ATR) module was used to characterize the polymer. The UV-visible spectra were measured using a Cary 1100 UV-Vis-IR spectrometer. Raman spectra were recorded by a Bruker Senterra Raman microscope using $\times 50$ objective with a laser excitation of $532 \mathrm{~nm}$. 


\section{Results and discussion}

Figure 1 shows the cyclic voltammetry of the electropolymerization reaction of $0.2 \mathrm{M}$ benzene, $0.2 \mathrm{M}$ thiophene, and their mixtures in [HMIM]FAP on copper at room temperature. From Fig. 1a, a sharp rise in current is observed at $1.45 \mathrm{~V}$ in the first $\mathrm{CV}$ cycle which relates to the oxidative polymerization of thiophene. In subsequent cycles, two small oxidation peaks around $1 \mathrm{~V}$ and $1.5 \mathrm{~V}$ are seen followed by a rise in current from $1.9 \mathrm{~V}$. The first two peaks have been ascribed to doping/ion movement of the polymer $[17,18]$ followed by further polymerization. The dedoping of the polymer is seen at 1.5 and $0.5 \mathrm{~V}$ [17].

On addition of different concentrations of benzene to the electrolyte, changes in the $\mathrm{CV}$ are observed (Fig. 1b-d). The copolymerization process occurs at potentials above $1.0 \mathrm{~V}$, and the doping/dedoping processes are not very prominent. Figure $1 \mathrm{e}$ shows the polymerization of benzene on $\mathrm{Cu}$ which starts at $1.8 \mathrm{~V}$. Subsequent cycles show that the polymerization process has additional oxidation peaks between 1.25 and $1.75 \mathrm{~V}$ which might be related to doping of PPP [17].

Figure 2a shows the low magnification SEM image of polythiophene electrodeposited on copper which shows the formation of nanoparticles over the entire substrate with a particle size of about $300 \mathrm{~nm}$. On copolymerization with PPP with 3:1 ratio of thiophene to benzene in the electrolyte, a highly nanoporous/microporous structure with porosities between $100 \mathrm{~nm}$ and $2.5 \mu \mathrm{m}$ is obtained (Fig. 2b, Fig. S1a). With 1:1 ratio of thiophene to benzene in the electrolyte, hierarchical porous polymer structures are observed with the presence of both nanopores and micropores ranging between $20 \mathrm{~nm}$ and
$7 \mu \mathrm{m}$ (Fig. 2c, Fig. S1b). On increasing the concentration of benzene in the electrolyte, polymer films with fewer pores are observed in Fig. 2d with a pore size of few micrometers (Fig. S1c), whereas polymer films with dents are observed on electrodeposited PPP (Fig. 2e). Thus, from the SEM results, it is evident that an optimum ratio of thiophene and benzene is needed to synthesize a hierarchical porous electrode which would create abundant catalytic sites for HER and minimize the hydrogen buildup.

X-ray photoelectron spectroscopy (XPS), FTIR, and UVvisible spectroscopy were performed to get an insight into the copolymerized deposit and to assess the semiconducting properties of the polymers. The high resolution XPS spectra of C $1 \mathrm{~s}$ are shown in Fig. 3a from which four peaks can be deconvoluted. For polythiophene, the peak at $284.7 \mathrm{eV}$ can be ascribed to $\mathrm{C}=\mathrm{C}-\mathrm{C}$, whereas the shoulder at $286.6 \mathrm{eV}$ is related to $\mathrm{C}=\mathrm{C}-\mathrm{S}$ [19]. Two shake-up satellite peaks are also observed at 289.6 and $292.8 \mathrm{eV}$ which indicate $\pi-\pi^{*}$ transitions in PTh valence electrons [20]. The change in intensity and position of the shake-up structures has been related to the change in the electronic structure of the polymer [21]. On addition of PPP into polythiophene with a ratio of 1:3 (benzene and thiophene), a clear change in intensity of these shake-up peaks is observed in Fig. 3a, which can be related to the change in the energy of molecular orbitals in the copolymer. The shoulder at $286.6 \mathrm{eV}$ observed for PTh almost forms a peak which is related to the change in the $\mathrm{C}=\mathrm{C}-\mathrm{S}$, thereby confirming the formation of a copolymer.

With 1:1 of benzene to thiophene, the $\mathrm{C}=\mathrm{C}-\mathrm{S}$ is significantly reduced but reappears on further increase in PPP concentration. However, a clear shift in the $\mathrm{C} 1 \mathrm{~s}$ peak and the


Fig. 1 CV of a $0.2 \mathrm{M}$ thiophene in HMIMFAP, b 0.15 M thiophene + 0.05 M benzene in HMIMFAP, $\mathbf{c} 0.1 \mathrm{M}$ thiophene $+0.1 \mathrm{M}$ benzene in HMIMFAP, d $0.05 \mathrm{M}$ thiophene $+0.15 \mathrm{M}$ benzene in HMIMFAP, and e $0.2 \mathrm{M}$ benzene in HMIMFAP 




Fig. 2 SEM of electrodeposited a PTh, b PTh + PPP from 3:1 ratio of thiophene to benzene in [HMIM]FAP, $\mathbf{c}$ PTh + PPP from 1:1 ratio of thiophene to benzene in [HMIM]FAP, $\mathbf{d}$ PTh + PPP from 1:3 ratio of thiophene to benzene in [HMIM]FAP, and e PPP

shake-up structures is observed in PPP. The main C 1s peak for PPP is centered at $285 \mathrm{eV}$ and is consistent with literature for $\mathrm{C}-\mathrm{C}$ [22]. A shoulder at $287.2 \mathrm{eV}$ is observed which could be assigned to $\mathrm{C}-\mathrm{O}$. The shake-up structures occur at 290 and $293.3 \mathrm{eV}$ which clearly indicate that the energy levels of molecular orbitals are different in PPP compared with the copolymers. The $\mathrm{S} 2 \mathrm{p}$ high resolution spectra are shown in Fig. $3 \mathrm{~b}$. It was observed that not all copolymers showed the presence of sulfur and the intensity of sulfur was very low for the copolymers. The S $2 p$ in PTh is composed of two wellseparated components at $163.8 \mathrm{eV}$ and $165 \mathrm{eV}$ which correspond to spin-orbital splitting [20]. A change in the ratio of the intensities in the copolymer is a clear indication for the formation of other bonds. FTIR was used to understand the changes in the stretching modes which occur in the copolymer. In Fig. 3c, it is evident that changes in $\mathrm{C}-\mathrm{H}$ stretching mode occur in PTh on copolymerization. Appearance of additional peaks between 1000 and $1200 \mathrm{~cm}^{-1}$ is also observed which confirms the formation of copolymers. The changes in $\mathrm{C}-\mathrm{H}$ in plane, out of plane, and terminal stretching modes are shown in Fig. 3d. As we go from PTh towards PPP, a blue shift from 789 to $804 \mathrm{~cm}^{-1}$ occurs in $\mathrm{C}-\mathrm{H}$ out of plane, whereas a red shift from 723 to $696 \mathrm{~cm}^{-1}$ occurs for $\mathrm{C}-\mathrm{H}$ terminal bonds. Peak broadening and peak splitting are also observed in Fig. $3 \mathrm{c}$ for the out of plane $\mathrm{C}-\mathrm{H}$ stretching which is a clear indication for the formation of copolymers. The UV-visible spectra of the conducting polymers are shown in Fig. 3e. Polythiophene shows an absorbance peak centered at $570 \mathrm{~nm}$ and a shoulder at $470 \mathrm{~nm}$ (black line, Fig. 3e), whereas PPP shows a wide absorbance band centered at $465 \mathrm{~nm}$ (green line, Fig. 3e). For both polymers compared with other synthesis routes [23-25], the electrochemical synthesis in ionic liquid shows much higher absorbance over a wide visible region. On copolymerizing PTh with PPP at 3:1 and 1:1 ratio of benzene to thiophene in the electrolyte (red and blue line, Fig. 3e), an increase in the absorbance and a red shift occurs from 570 to $593 \mathrm{~nm}$. A blue shift is also seen from 470 to $400 \mathrm{~nm}$. However, with 1:3 ratio of benzene to thiophene, a peak at 580 and a shoulder at $502 \mathrm{~nm}$ is observed. Thus, it is evident from the spectroscopic measurements that copolymerization has indeed occurred and the UV spectra show a wide absorbance of the copolymers in the visible range. These blue and red shifts indicate a change in the HOMO/LUMO levels in the copolymers compared with PPP and PTh.

Before testing the as-synthesized polymers/copolymers for hydrogen evolution reaction (HER) in $1 \mathrm{M} \mathrm{KOH}$ under dark and visible conditions, we investigated the contact angles of an alkaline solution on the polymers, as wettability affects the HER reaction [26, 27]. We first measured the contact angles of the synthesized polymers/copolymers. The wettability of the polymer in $1 \mathrm{M} \mathrm{KOH}$ is shown in Fig. 4a. PTh and PPP show a contact angle of 53 and $72^{\circ}$, respectively (Fig. 4b). Among the copolymers, the lowest contact angle was found for 1:1 PTh:PPP composition with a value of $22^{\circ}$ (Fig. 4b). The change in wettability can be related to a change in polymer structure as evident from IR spectra as well as change in the porosity of the polymer as seen from SEM. As the wettability is highest for 1:1 composition, the hydrogen evolution can be expected at a lower overpotential. Figure $4 \mathrm{c}$ and $\mathrm{d}$ show the HER on different polymers in dark and under visible light, respectively. Under dark conditions (Fig. 4c), copolymers always had a lower overpotential compared with individual 

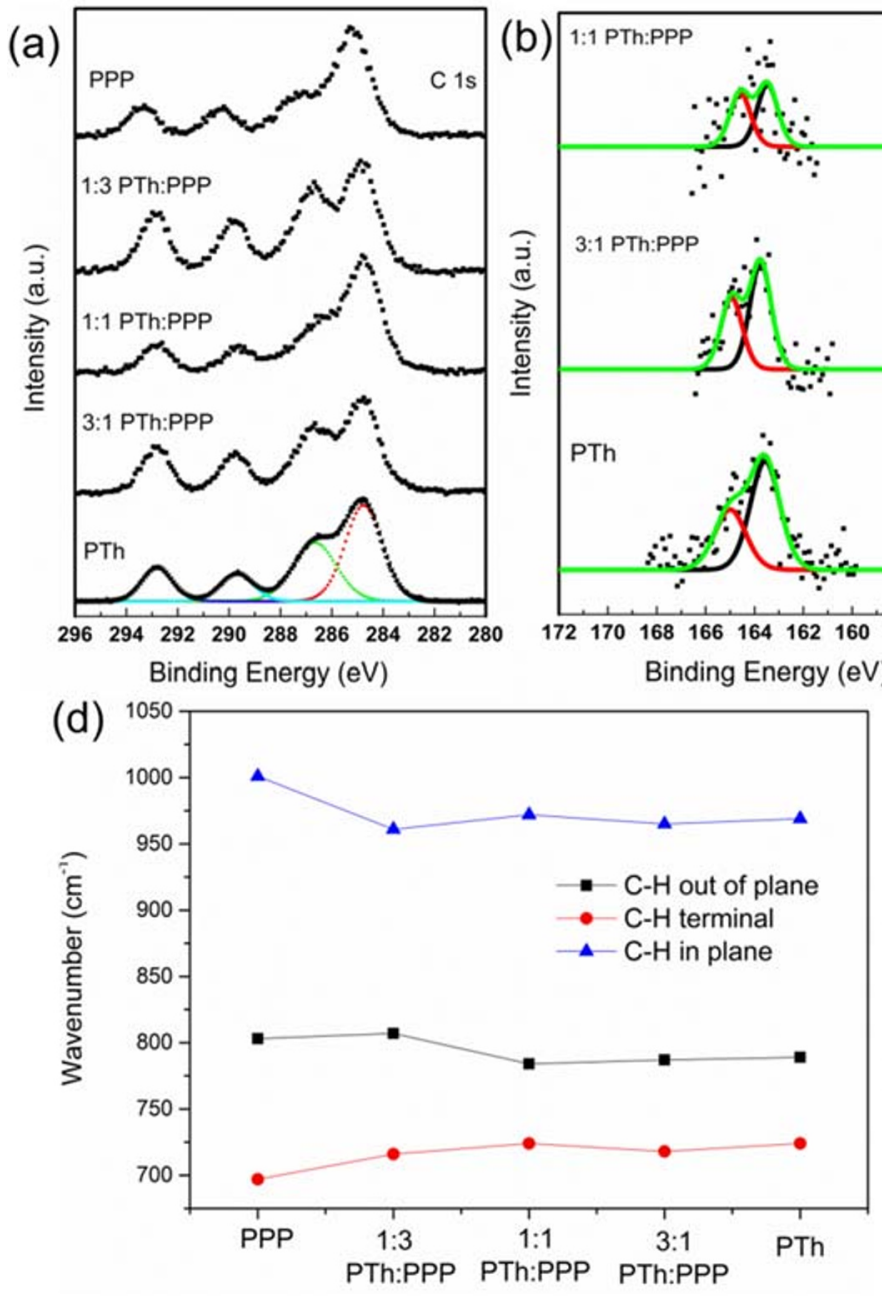
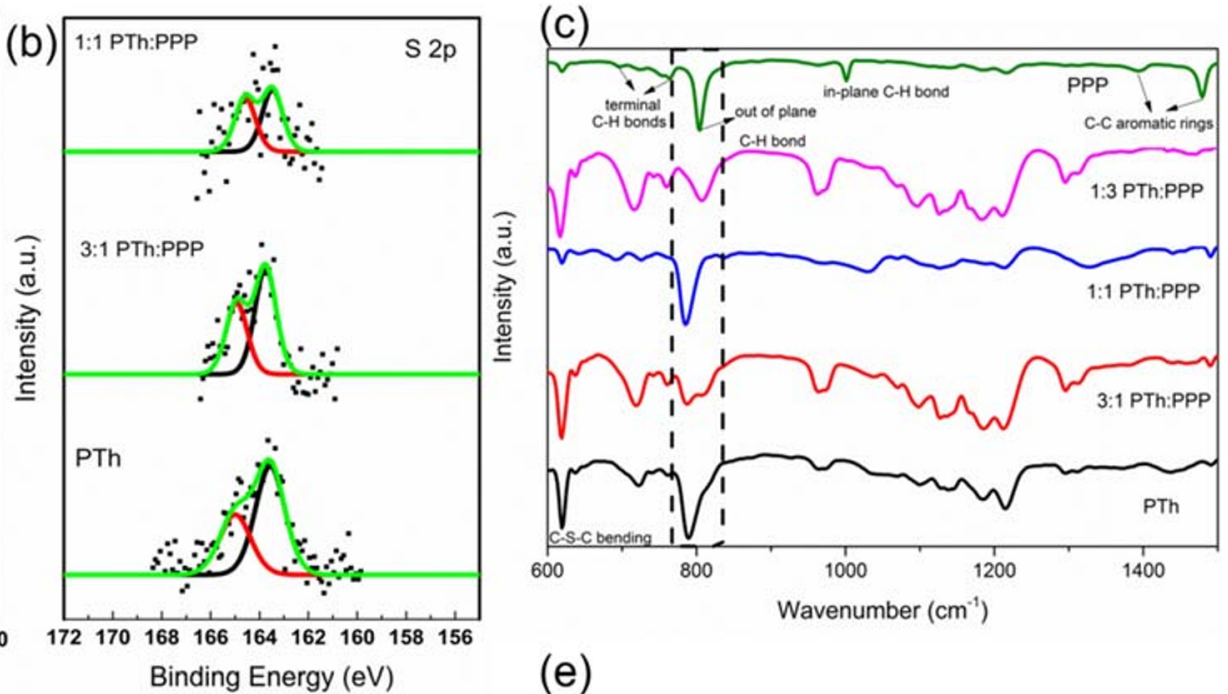

(e)



Fig. 3 a High resolution XPS of C 1s of different polymers. b XPS of S 2p of different polymers. $\mathbf{c}$ FTIR spectra of PPP and PPP:PTh copolymers. d Change in the $\mathrm{C}-\mathrm{H}$ stretching in PPP and PPP:PTh copolymers. e UV-visible spectroscopy of different polymers and copolymers

polymers, and an overpotential of $455 \mathrm{mV}$ was required to obtain a current density of $10 \mathrm{~mA} \mathrm{~cm}{ }^{-2}$ for both $1: 1$ PTh:PPP and 1:3 PTh:PPP copolymers. In comparison, the experiments performed under visible light showed much higher current densities at lower overpotentials for HER (Fig. 4d) which indicates that a light-induced charge transfer process might be taking place.

Interestingly, for a current density of $10 \mathrm{~mA} \mathrm{~cm}^{-2}$, a lowest overpotential of $417 \mathrm{mV}$ vs RHE was observed under visible light for copolymers having 3:1 PTh:PPP composition which is comparatively lower with other polymeric materials mentioned in literature $[28,29]$. The overpotential of both $1: 1$ and 1:3 PTh:PPP was found to be $427 \mathrm{mV}$ to obtain a current density of $10 \mathrm{~mA} \mathrm{~cm}^{-2}$ (Fig. 4d). Therefore, a direct relationship between wettability at open circuit potential and electrocatalysis is not that simple, and one needs to look into electro-wettability and three-phase interface of the polymer, $\mathrm{KOH}$ and hydrogen production, and also the electronic structure of the catalysts. All polymers/copolymers except PPP obtained higher current densities at more or less similar overpotentials in the presence of light, whereas only $1: 1$ PTh:PPP and 1:3 PTh:PPP copolymers achieved $10 \mathrm{~mA} \mathrm{~cm}^{-2}$ under dark conditions.

Heat treatment of conducting polymers has shown to affect the conductivity as well as to change its structure $[30,31]$. Upon heat treatment, a remarkable shift in the overpotential and current density was observed for all the polymers. The microstructure after heat treatment (Fig. S2) showed that all the polymers have an open network structure. The XPS of heat-treated samples showed certain changes in $\mathrm{C} 1 \mathrm{~s}$ and $\mathrm{S}$ 2p (Fig. 5a and b) spectra. For PTh, 3:1 PTh:PPP and 1:1 PTh:PPP (Fig. 5a), the carbon peak at $284.7 \mathrm{eV}$ (Fig. 5a) related to $\mathrm{C}=\mathrm{C}-\mathrm{C}$ does not change upon heat treatment, whereas the shoulder at $286.6 \mathrm{eV}$ related to $\mathrm{C}=\mathrm{C}-\mathrm{S}$ drastically decreases compared to that observed in Fig. 3a. Furthermore, the shake-up structures which occurred at 290 and $293.3 \mathrm{eV}$ have (Fig. 3a) disappeared. As these peaks are related with energy levels of molecular orbitals, it appears that carbonization of the polymers took place which resulted in the loss of semiconducting property of the polymer. However, the 
(a)



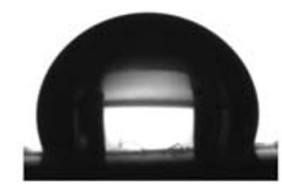

PTh:PPP 3:1

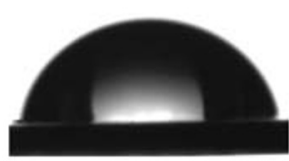

PPP
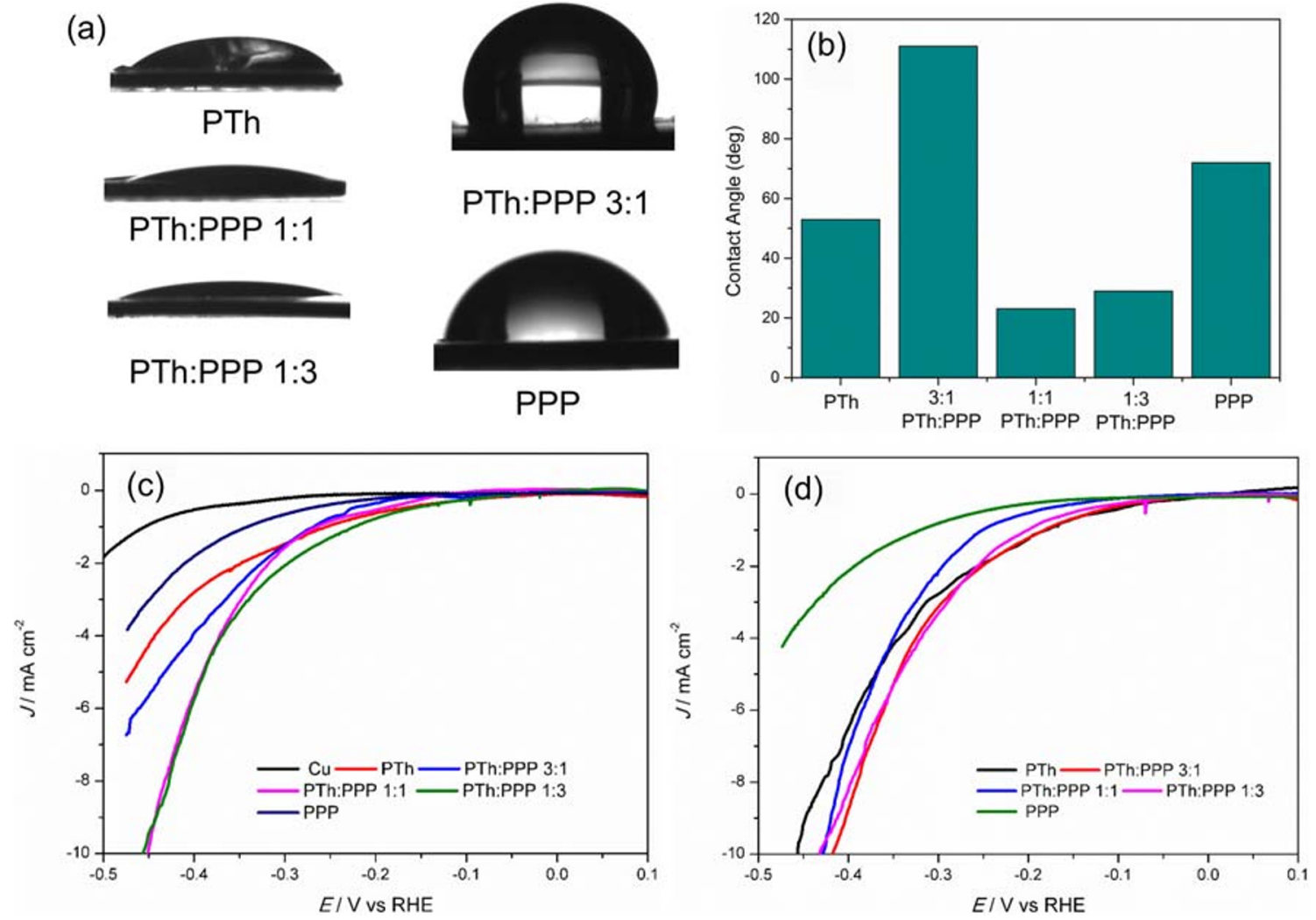

Fig. 4 a, b Surface contact angle measurement for different polymers in $1 \mathrm{M} \mathrm{KOH}$. $\mathbf{c}$ HER on different polymers under dark and $\mathbf{d}$ under visible light conditions in $1 \mathrm{M} \mathrm{KOH}$ at a scan rate of $10 \mathrm{mV} \mathrm{sec}^{-1}$

presence of sulfur in the heat-treated polymer (Fig. 5b) indicates that full carbonization of the polymer might not have taken place. For the case of PPP and 1:3 PTh:PPP, both the peaks at $284.7 \mathrm{eV}$ and the shoulder at $286.6 \mathrm{eV}$ are prominent. Also, a small satellite peak at $293 \mathrm{eV}$ is observed which indicates that these polymers still retain their semiconducting property with a few changes in their electronic structure. It was reported that the carbonization of polythiophene at $700{ }^{\circ} \mathrm{C}$ has shown to improve the electrical conductivity [30], whereas heat treatment of PPP leads to a change in the structure from a benzenoid to a defect-quinoid structure [31].

Therefore, from XPS measurements, it is clear that the orbital structure of the polymers is affected on heat treatment which improved the HER electrocatalytic property of the materials. The change in the polymer structure and the electronic structure is further substantiated by IR and UV spectra. From the IR spectra in Fig. S3a, both PPP and 1:3 PTh:PPP copolymers show only the $\mathrm{C}-\mathrm{H}$ stretching modes, whereas these modes are completely lost for polythiophene and the other two copolymers with higher thiophene content. The Raman spectra of heat-treated polymers are compared in Fig. S3b. PTh shows the $\mathrm{D}$ and $\mathrm{G}$ bands at 1342 and $1573 \mathrm{~cm}^{-1}$, respectively, which correspond well to the formation of graphite/graphene mixture. However on increasing the PPP concentration (1:3 PTh:PPP), a clear shift in the $\mathrm{G}$ band to $1598 \mathrm{~cm}^{-1}$ and $\mathrm{D}$ band to $1331 \mathrm{~cm}^{-1}$ with an additional shoulder at $1236 \mathrm{~cm}^{-1}$ occur which are related to the defect-quinoid structure of PPP [31]. Furthermore, the heat-treated PPP clearly shows the defectquinoid structure of PPP and does not correspond to the formation of graphite. The changes in the stretching modes along with the formation of defects affect the electronic structure of the polymers which were observed by the changes in the UVvisible spectra (Fig. S3c). A lowering of absorption along with the blue shift in the absorption from 590 to $560 \mathrm{~nm}$ and 400 to $300 \mathrm{~nm}$ intensity is observed for all the polymers. Such shifts clearly represent a change in the electronic band structure of the partially carbonized polymers.

Heat treatment also resulted in a change in wetting behavior. Figure $5 \mathrm{c}$ shows the wetting behavior of $1 \mathrm{M} \mathrm{KOH}$ after heat treatment. From the contact angle measurements of the heat-treated polymers, besides PPP, all other polymers show a contact angle below $30^{\circ}$ with a complete wetting for 1:1 PTh:PPP. The HER plots in Fig. 5d show a remarkable shift in the catalytic performance. The asprepared PPP which could not achieve a high current density before heat treatment showed a remarkable HER activity by achieving a current density of $10 \mathrm{~mA} \mathrm{~cm}^{-2}$ at quite a low overpotential of $105 \mathrm{mV}$ upon heat treatment. Both PTh and PTh:PPP 1:3 also showed a significant HER performance which commenced at around $35 \mathrm{mV}$ and achieved a current density of $10 \mathrm{~mA} \mathrm{~cm}{ }^{-2}$ at 110 and $85 \mathrm{mV}$ overpotential, respectively. An unprecedented low 

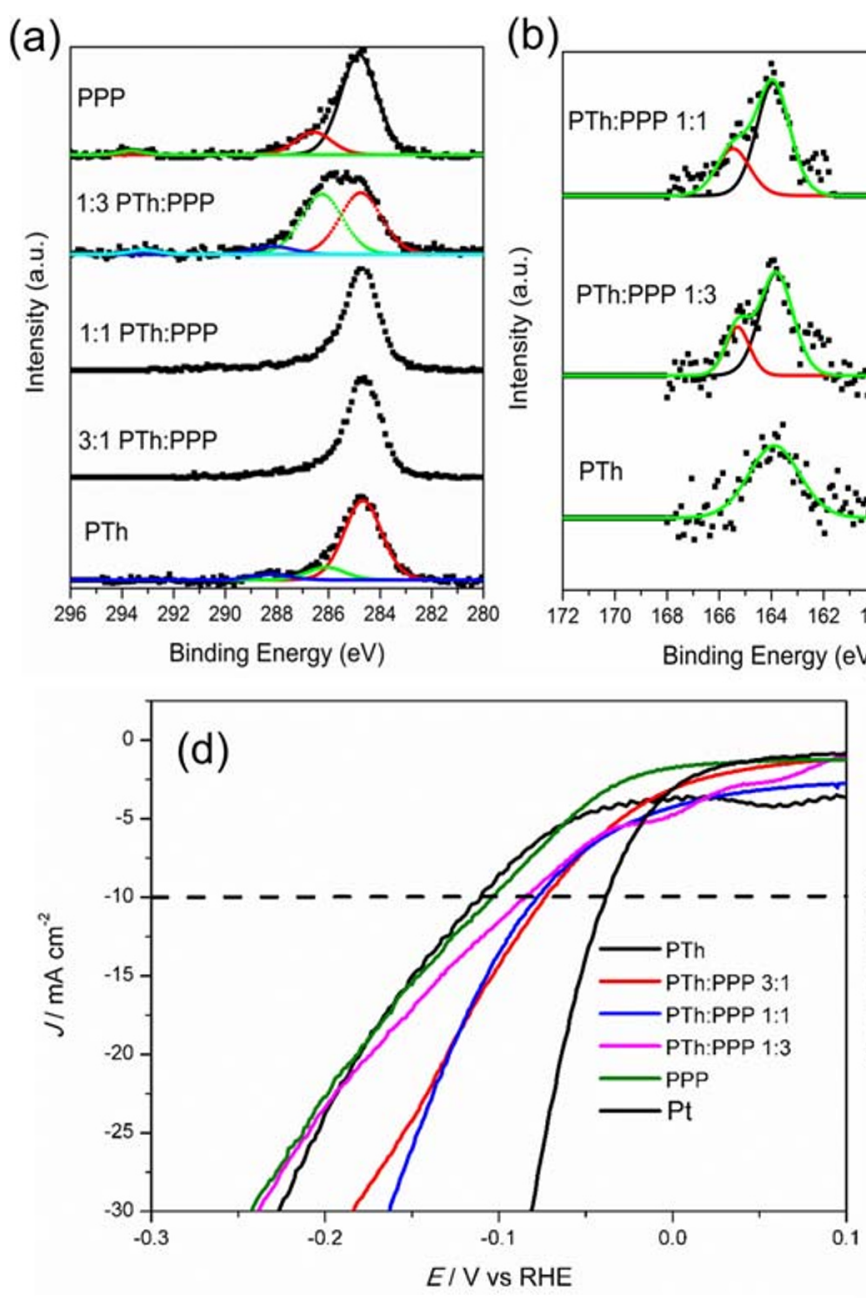

Fig. 5 a High resolution XPS of C $1 \mathrm{~s}$ of different polymers. b XPS of S $2 \mathrm{p}$ of different polymers after heat treatment at $700^{\circ} \mathrm{C}$ for $1 \mathrm{~h}$. $\mathbf{c}$ Contact angle measurement of heat-treated PPP and PPP:PTh copolymers with

overpotential of just $77 \mathrm{mV}$ was required to achieve a current density of $10 \mathrm{~mA} \mathrm{~cm}^{-2}$ for both 1:1 PTh:PPP and 3:1 PTh:PPP copolymers (Fig. 5d). Repeated experiments showed that the HER overpotential values for both the polymers were in the range of $\sim 75-90 \mathrm{mV}$ to obtain a current density of $10 \mathrm{~mA} \mathrm{~cm}{ }^{-2}$ which might be due to the difference in the polymer structure and porosity in the (b)

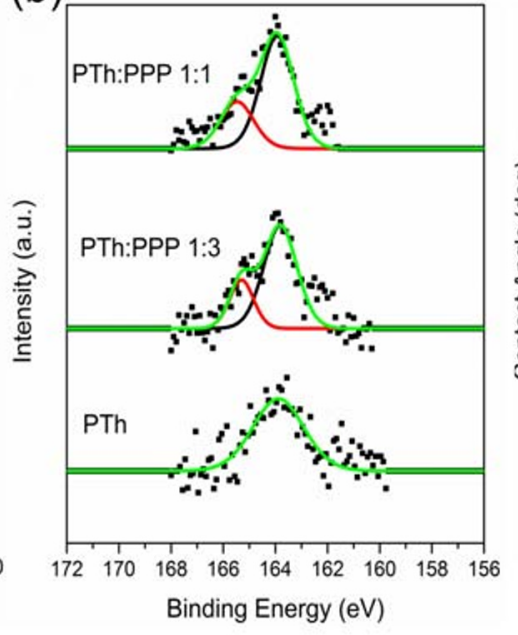

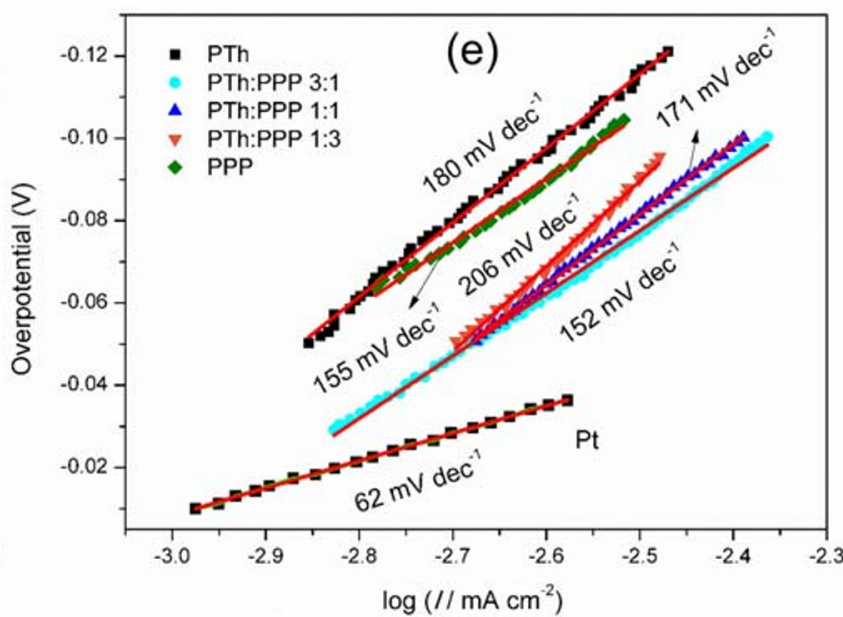

different ratios at $700{ }^{\circ} \mathrm{C}$. d HER catalysis on different heat-treated polymers. e Tafel plots of heat-treated polymers

polymer. Table 1 compares the HER of the heat-treated polymer with metal-free catalyst and carbon-based catalyst. It is evident that the results obtained in this work show the lowest overpotential to reach a current density of $10 \mathrm{~mA} \mathrm{~cm}^{-2}$.

The HER values are slightly higher than Pt which shows an overpotential of $37 \mathrm{mV}$ to achieve a current density of

Table 1 Comparison of HER performance on different metal-free catalysts in $1 \mathrm{M} \mathrm{KOH}$

\begin{tabular}{lllll}
\hline Material & Method & $\mathrm{H}\left(\mathrm{V}\right.$ vs RHE) $J=-10 \mathrm{~mA} \mathrm{~cm}{ }^{-2}$ & Tafel slope $\left(\mathrm{mV}\right.$ dec $\left.^{-1}\right)$ & Reference \\
\hline N, P codoped carbon network & Pyrolysis & 0.16 & 89 & {$[32]$} \\
3D porous carbon & Polymerization + pyrolysis & 0.446 & 154 & 79 \\
3D carbon network & Pyrolysis & 0.131 & 116.7 & {$[33]$} \\
N-doped graphene & Pyrolysis & 0.432 & 112 & {$[34]$} \\
N, S codoped graphitic sheet & Lyophilization and pyrolysis & $0.31(0.1 \mathrm{M} \mathrm{KOH})$ & 171 & {$[35]$} \\
This work & Electropolymerization + pyrolysis & 0.077 & & \\
\hline
\end{tabular}

Electrolyte differing from $1 \mathrm{M} \mathrm{KOH}$ has been shown in brackets 
$10 \mathrm{~mA} \mathrm{~cm}{ }^{-2}$. Furthermore, compared with precious noble metal nanoparticles and their carbon composites [37], the overpotential achieved by the porous polymer is higher by about $50-70 \mathrm{mV}$.

The Tafel plots for HER for different polymers are plotted in Fig. 5e using the Tafel equation as shown in Eq. 1.

$\eta=a+b \cdot \log |j|$.

In Eq. 1, $\eta$ is the overpotential which is the difference between the electrode and standard electrode potential $(\eta=$ $\left.\mathrm{E}-\mathrm{E}_{\mathrm{o}}\right), j$ denotes the current density, and $b$ is the Tafel slope. All the polymers showed a Tafel slope between 150 and $205 \mathrm{mV} \mathrm{dec}^{-1}$. Based on the Tafel-Volmer-Heyrovsky mechanism for HER in alkaline medium, the rate determining step for HER is the Volmer and Tafel steps which is related with the hydrogen adsorption and hydrogen reduction, respectively. In the case of the polymers, it is evident that the Tafel slopes are higher than $120 \mathrm{mV} \mathrm{dec}^{-1}$ which indicates that electrochemical formation of adsorbed hydrogen atoms is the limiting step and controls the kinetics of catalysis [38-40]. With suitable modification by incorporating metal nanoparticles, it might be possible to lower the Tafel slope values and increase the rate of hydrogen evolution at the electrode surface. HER experiments were also performed on 1:1 PTh:PPP copolymer over time at $90 \mathrm{mV}$ overpotential vs RHE (Fig. S4a) which showed an initial increased current density of greater than $15 \mathrm{~mA} \mathrm{~cm}^{-2}$ for the first $5 \mathrm{~h}$ and stabilized at a current density of $10 \mathrm{~mA} \mathrm{~cm}^{-2}$ for $30 \mathrm{~h}$. The disturbance in the current density measurement was due to the hydrogen evolution at the porous electrode surface. After $30 \mathrm{~h}$ electrolysis, the HER performance of the polymer was again tested which showed almost the same performance as before electrolysis (Fig. S4b). Thus, based on the presented results, it appears that heat treatment of polymers not only changes the electronic structure but also improves the conductivity, porosity, and the wetting behavior of the polymers, all of which contributes to enhance the catalytic properties of polymers and lower the HER overpotentials significantly.

\section{Conclusion}

In conclusion, we have shown a simple electrochemical strategy to obtain porous conducting polymers which show a high catalytic property for HER. The copolymerization leads to a change in the electronic structure and improves the absorption of light. In the presence of visible light, the lowest overpotential of $427 \mathrm{mV}$ is required to obtain $10 \mathrm{~mA} \mathrm{~cm}{ }^{-2}$ for 3:1 PTh:PPP. Heat treatment remarkably lowers the HER on all the polymers. The lowest overpotential of only $77 \mathrm{mV}$ was required to achieve a current density of $10 \mathrm{~mA} \mathrm{~cm}^{-2}$ for both 1:1 PTh:PPP and 3:1 PTh:PPP copolymers. The polymers were also shown to be stable for hydrogen production over long time with little deterioration in HER performance. Thus, here, we have shown a simple approach to obtain highly porous polymeric materials with tunable absorbance and high electrocatalytic activity which paves new avenues for exploring these metal-free electrodes for other energy conversion applications.

Funding Information Open Access funding provided by Projekt DEAL.

Open Access This article is licensed under a Creative Commons Attribution 4.0 International License, which permits use, sharing, adaptation, distribution and reproduction in any medium or format, as long as you give appropriate credit to the original author(s) and the source, provide a link to the Creative Commons licence, and indicate if changes were made. The images or other third party material in this article are included in the article's Creative Commons licence, unless indicated otherwise in a credit line to the material. If material is not included in the article's Creative Commons licence and your intended use is not permitted by statutory regulation or exceeds the permitted use, you will need to obtain permission directly from the copyright holder. To view a copy of this licence, visit http://creativecommons.org/licenses/by/4.0/.

\section{References}

1. Mahmood N, Yao Y, Zhang JW, Pan L, Zhang X, Zou JJ (2018) Electrocatalysis for hydrogen evolution in alkaline electrolytes: mechanisms, challenges, and prospective solutions. Adv Sci 5: 1700464/1-1700464/23

2. Giles RD, Harrison JA, Thirsk HR (1969) Catalytic hydrogen evolution on ruthenium and platinum nuclei. J Electroanal Chem 20: $47-60$

3. Sheng W, Gasteiger HA, Shao-Horn Y (2010) Hydrogen oxidation and evolution reaction kinetics on platinum: acid vs alkaline electrolytes. J Electrochem Soc 157:B1529-B1536

4. Wang J, Xu F, Jin H, Chen Y, Wang Y (2017) Non-noble metalbased carbon composites in hydrogen evolution reaction: fundamentals to applications. Adv Mater 29:1605838/1-1605838/35

5. Vesborg PCK, Seger B, Chorkendoff I (2015) Recent development in hydrogen evolution reaction catalysts and their practical implementation. J Phys Chem Lett 6(6):951-957

6. Merki D, Hu X (2011) Recent developments of molybdenum and tungsten sulfides as hydrogen evolution catalysts. Energy Environ Sci 4:3878-3888

7. Zou X, Zhang Y (2015) Noble metal-free hydrogen evolution catalysts for water splitting. Chem Soc Rev 44(15):5148-5180

8. Zheng Y, Jiao Y, Zhu Y, Li LH, Han Y, Chen Y, Du A, Jaroniec M, Qiao SZ (2014) Hydrogen evolution by a metal-free catalyst. Nat Commun 5:3783/1-3783/8

9. Hu C, Dai L (2019) Doping of carbon materials for metal-free electrocatalysis. Adv Mater 31:1804672/1-1804672/17

10. Zhou Q, Shi G (2016) Conducting polymer-based catalysts. J Am Chem Soc 138(9):2868-2876

11. Yao L, Rahmanudin A, Guijarro N, Sivula K (2018) Organic semiconductor based devices for solar water splitting. Adv Energy Mater 8:1802585/1-1802585/18 
12. Winter-Jensen B, Fraser K, Ong C, Forsyth M, MacFarlane DR (2010) Conducting polymer composite materials for hydrogen generation. Adv Mater 22:1727-1730

13. Wu D, Xu F, Sun B, Fu R, He H, Matyjaszewski K (2012) Design and preparation of porous polymers. Chem Rev 112(7):3959-4015

14. Biallozor S, Kupniewska A (2005) Conducting polymers electrodeposited on active metals. Syn Metals 155:443-449

15. Snook GA, Greaves TL, Best AS (2011) A comparative study of electrodeposition of polyaniline from a protic ionic liquid, an aprotic ionic liquid and neutral aqueous solution using anilinium, nitrate. J Mater Chem 21:7622-7629

16. Carstens T, Zein El Abedin S, Endres F (2008) Electrosynthesis of poly(para)phenylene in an ionic liquid: cyclic voltammetry and in situ STM/tunneling spectroscopy studies. ChemPhysChem 9(3): 439-444

17. Pringle JM, Forsyth M, MacFarlane DR, Wagner K, Hall SB, Officer DL (2005) The influence of the monomer and the ionic liquid on the electrochemical preparation of polythiophene. Polymer 46:2047-2058

18. Reynolds JR, Hsu SG, Arnott HJ (1989) The effect of electrolytecontrolled growth morphology on the charge transport properties of poly(3-methylthiophene). J Poly Sci B Poly Phys 27:2081-2103

19. Heeg J, Kramer C, Wolter M, Michaelis S, Plieth W, Fischer WJ (2001) Polythiophene- $\mathrm{O}_{3}$ surface reactions studied by XPS. App Surf Sci 180:36-41

20. Tourillon G, Jugnet Y (1988) Electronic and structural characteristics of five poly membered heterocycles (polythiophene, polypyrrole): an ultraviolet and X-ray photoelectron spectroscopy study. J Chem Phys 89:1905-1913

21. Jardella JA, Ferguson SA, Chin RL (1986) $\pi^{*} \leftarrow \pi$ shakeup satellites for the analysis of structure and bonding in aromatic polymer by X-ray photoelectron spectroscopy. Appl Spectroscopy 40:224 232

22. Nguyen TP, Ettaik H, Lefrant S, Lessing G, Stelzer F (1990) Studies of the polyparaphenylene/aluminium interface. Synth Met 38:6976

23. Mulazzi E, Ripamonti A, Athouël L, Wery J, Lefrant S (2002) Theoretical and experimental investigation of the optical properties of poly(paraphenylene): evidence of chain-length distribution. Phys Rev B 65:085204/1-085204/9

24. Shao M, He Y, Hong K, Rouleau CM, Geohegan DB, Xiao KA (2013) Water-soluble polythiophene for organic field-effect transistors. Poly Chem 4:5270-5274

25. Clément S, Tizit A, Desbief S, Mehdi A, De Winter J, Gerbaux P, Lazzaroni R, Boury B (2011) Synthesis and characterisation of $\pi$ conjugated polymer/silica hybrids containing regioregular ionic polythiophenes. J Mater Chem 21:2733-2739

26. Gao L, Qian SY, Conway BE (1995) Electrolyte wetting effects in the comparative polarization behavior of the hydrogen evolution reaction at Ni-Mo-Cd electrodes in KF.2HF and KOH. $2 \mathrm{H}_{2} \mathrm{O}$ melts. J Appl Electrochem 25:6-14
27. Wang L, Xiao FS (2014) The importance of catalyst wettability. ChenCatChem 6:3048-3052

28. Lai J, Li S, Wu F, Saqib M, Luque R, Xu G (2016) Unprecedented metal-free 3D porous carbonaceous electrodes for full water splitting. Energy Environ Sci 9:1210-1214

29. Downes CA, Marinescu SC (2017) Bioinspired metal selenolate polymers with tunable mechanistic pathways for efficient $\mathrm{H}_{2}$ evolution. ACS Catal 7:8605-8612

30. Ando E, Onodera S, Iino M, Ito O (2001) Electric conductivity changes of polypyrrole and polythiophene films with heat-treatment. Carbon 39:101-108

31. Matthews MJ, Brown SDM, Dresselhaus MS, Endo M, Takamuku T, Karaki T (1999) Optical properties of heat-treated polyparaphenylene. J Mater Res 14:1091-1101

32. Zhang J, Qu L, Shi G, Liu J, Chen J, Dai L (2016) N, P-Codoped carbon networks as efficient metal-free bifunctional catalysts for oxygen reduction and hydrogen evolution reaction. Angew Chem Int Ed 55:2230-2234

33. Lai J, Li S, Wu F, Saquib M, Lugue R, Xu G (2016) Unprecedented metal-free $3 \mathrm{D}$ porous carbonaceous electrodes for full water splitting energy. Environ Sci 9:1210-1214

34. Peng Z, Yang S, Jia D, Da P, He P, Al-Enizi AM, Ding G, Xie X, Zheng G (2016) Homologous metal-free electrocatalysts grown on three-dimensional carbon networks for overall water splitting in acidic and alkaline media. J Mater Chem A 4:12878-12883

35. Zhang B, Wang H-H, Su H, Lv L-B, Zhao T-J, Ge J-M, Wei X, Wang KX, Li XH, Chen JS (2016) Notrigen-doped graphene microtubes with opened inner voids: highly efficient metal-free electrocatalysts for alkaline hydrogen evolution reaction. Nano Res 9:2606-2615

36. $\mathrm{Hu} \mathrm{C}$, Dai L (2017) Multifunctional-carbon based metal-free electrocatalysts for simultaneous oxygen reduction, oxygen evolution, and hydrogen evolution. Adv Mater 29:1604942/1-1604942/ 9

37. Safizadeh F, Ghali E, Houlachi G (2015) Electrocatalysts developments for hydrogen evolution reaction in alkaline solutions - a review. Int J Hydrog Energy 40:256-274

38. Conway BE, Tilak BV (1992, chapter 1) In: Eley DD, Pines H, Weisz PB (eds) Advances in catalysis, vol 38. Academic Press, San Diego, pp 1-147

39. Conway BE, Tilak BV (2002) Interfacial processes involving electrocatalystic evolution and oxidation of $\mathrm{H} 2$, and the role of chemsorbed H. Electrochim Acta 47:3571-3594

40. Shinagawa T, Garcia-Esparza AT, Takanabe K (2015) Insight on Tafel slopes from a microkinetic analysis of aqueous electrocatalysis for energy conversion. Sci Rep 5:13801/1-13801/ 21

Publisher's note Springer Nature remains neutral with regard to jurisdictional claims in published maps and institutional affiliations. 